\title{
Corrigendum: The Application of Regulatory Cascades in Streptomyces: Yield Enhancement and Metabolite Mining
}

\author{
Haiyang Xia ${ }^{1}$, Xiaofang $\mathrm{Li}^{1}$, Zhangqun $\mathrm{Li}^{1}$, Xinqiao Zhan ${ }^{1}$, Xuming Mao ${ }^{1,2 *}$ and \\ Yongquan $L i^{1,2 *}$ \\ ${ }^{1}$ Institute of Biopharmaceuticals, Taizhou University, Taizhou, China, ${ }^{2}$ Institute of Pharmaceutical Biotechnology, School of \\ Medicine, Zhejiang University, Hangzhou, China
}

Keywords: antibiotic production, regulatory cascades, rewiring regulatory network, unlocking cryptic metabolites, Streptomyces

\section{A Corrigendum on}

The Application of Regulatory Cascades in Streptomyces: Yield Enhancement and Metabolite Mining

by Xia, H., Li, X., Li, Z., Zhan, X., Mao, X., and Li, Y. (2020). Front. Microbiol. 11:406. doi: 10.3389/fmicb.2020.00406

Edited by:

Yvonne Mast

German Collection of Microorganisms and Cell Cultures GmbH

(DSMZ), Germany

Reviewed by:

Guenther Muth,

University of Tübingen, Germany

*Correspondence: Xuming Mao

xmmao@zju.edu.cn

Yongquan $L$

lyq@zju.edu.cn

Specialty section:

This article was submitted to

Microbial Physiology and Metabolism,

a section of the journal

Frontiers in Microbiology

Received: 05 October 2020 Accepted: 15 December 2020

Published: 03 February 2021

Citation:

Xia H, Li X, Li Z, Zhan X, Mao X and

Li Y (2021) Corrigendum: The

Application of Regulatory Cascades in

Streptomyces: Yield Enhancement

and Metabolite Mining.

Front. Microbiol. 11:614274.

doi: 10.3389/fmicb.2020.614274
In the original article, there was some errors. A portion of this text was reproduced from another article, this has now been rephrased and appropriately attributed.

A correction has been made to The Regulatory Cascades of Antibiotic Production in Streptomyces, paragraph 5:

The fourth level is the feedback regulation which is brought by antibiotic and/or intermediates to coordinate antibiotic production and transport. Evidence has shown that antibiotic functions as signals to regulate the production of antibiotic besides as feedback substances for the enzymatic reactions. Antibiotic, as ligand for proper regulator, affects the final production in Streptomyces. The expression of antibiotic biosynthetic genes was modulated by the RedZ and undecylprodigiosin complex (Wang et al., 2009). The activity of AtrA, which regulates primary and secondary metabolism, is reduced by lidamycin of Streptomyces globisporus and actinorhodin (ACT) of S. coelicolor ( $\mathrm{Li}$ et al., 2015). The biosynthesis of jadomycin is dynamically modulated by the interaction among jadomycin B, chloramphenicol, JadR1 and JadR2 in Streptomyces venezuelae (Wang et al., 2009; Xu et al., 2010). Daunorubicin (DNR) biosynthesis is regulated by three DNA binding regulatory proteins (DnrI, DnrN, and DnrO). The DNA binding activity of DnrO can be modulated by Rhodomycin D, a glycosylated precursor of DXR (Jiang and Hutchinson, 2006). Simocyclinone and its precursors inhibit the binding activity of SimReg1 to several promoter regions of simocyclinone biosynthesis genes and SimReg1 encoding gene (Horbal et al., 2012). As a GBL receptor-like protein, PapR5, which is the major regulator of pristinamycin biosynthesis, may sense pristinamycin or intermediate(s) of the pathway (Mast et al., 2015). SsaA can activate sansanmycin biosynthesis by binding to five different regions within the sansanmycin BGC. The sansanmycins $\mathrm{A}$ and $\mathrm{H}$ inhibit DNA-binding activity of SsaA in a concentration-dependent manner (Li et al., 2013). The rifamycin B, the end product of rifamycin biosynthesis, can relieve the repression of RifQ on the transcription of the rifamycin efflux pump (RifP) (Lei et al., 2018). Transporters may affect product maturation. Deletion of $n y s G$ and $n y s H$, two ABC transporters encoding genes, resulted in ca. $35 \%$ reduction of nystatin production and accumulation of its deoxy 
precursor in Streptomyces noursei. NysGH complex is prone to export nystatin. Its activity would enhance the last biosynthetic step by relief of the feedback through final product removal (Sletta et al., 2005). 'LanT, the dedicated ABC transporter for both class I and II lantibiotics, plays an important role in production of the final product (Gebhard, 2012).

A correction has been made to Enhancing Antibiotic Production by Overexpression of Positive Regulator Genes, paragraph 1:

The regulators also can be defined as positive and negative regulators according their effect on the antibiotic production. The positive regulators (activators) can promote the biosynthesis of antibiotics. But the negative ones (repressors) can repress the biosynthesis of antibiotics (Martin and Liras, 2010). Since the positive regulators activate the transcription of antibiotic BGCs, they can be manipulated to enhance the production of antibiotic in Streptomyces. The titer improvement can efficiently and simply be achieved by over-expression of genes encoding activators with proper promoters. As listed in Table 1, overexpression of genes encoding LAL family regulators, such as MilR, NemR, and AveR, has been used to increase production of milbemycin in S. bingchenggensis BC04, nemadectin in S. cyaneogriseus subsp. non-cyanogenus NMWT1 and avermectin in S. avermitilis, respectively (Guo et al., 2010; Zhang et al., 2016; Li et al., 2019). Overexpression of $\operatorname{san} G$, a CSR activator encoding gene, led to improvement of nikkomycin production (Liu et al., 2005). Tandem copies of otcR (a CSR activator gene), whose expression is driven by the SF14 promoter, can greatly enhance the production of oxytetracycline (OTC) (Yin et al., 2015). There are many examples in similar strategies to improve antibiotic production in Streptomyces. Overexpression of bulZ, fkbR1, $w y s R$, and $\ln m O$ led to overproduction of tacrolimus (FK506), ascomycin, wuyiencin, and leinamycin, respectively (Liu et al., 2014; Huang et al., 2016; Song et al., 2017; Ma et al., 2018).

A correction has been made to Enhancing Antibiotic Production by Manipulation of Feedback and Transport, Paragraph 1:

Genes encoding exporters, which are responsible for the secretion of antibiotic, often situate in their BGCs. Various BGC-linked transporters, belonging to ATP-binding cassette

\section{REFERENCES}

Foulston, L. C., and Bibb, M. J. (2010). Microbisporicin gene cluster reveals unusual features of lantibiotic biosynthesis in actinomycetes. Proc. Natl. Acad. Sci. U.S.A. 107, 13461-13466. doi: 10.1073/pnas.1008 285107

Gebhard, S. (2012). ABC transporters of antimicrobial peptides in Firmicutes bacteria - Phylogeny, function and regulation. Mol. Microbiol. 86, 1295-1317. doi: $10.1111 / \mathrm{mmi} .12078$

Guo, J., Zhao, J., Li, L., Chen, Z., Wen, Y., and Li, J. (2010). The pathway-specific regulator AveR from Streptomyces avermitilis positively regulates avermectin production while it negatively affects oligomycin biosynthesis. Mol. Genet. Genomics 283, 123-133. doi: 10.1007/s00438-009-0502-2

Horbal, L., Rebets, Y., Rabyk, M., Makitrynskyy, R., Luzhetskyy, A., Fedorenko, V., et al. (2012). SimReg1 is a master switch for biosynthesis and export of simocyclinone D8 and its precursors. AMB Express. 2:1. doi: $10.1186 / 2191-0855-2-1$
(ABC) superfamily and major facilitator superfamily (MFS) are responsible for secreting antibiotics. Pumping out of toxic endproducts can achieve more durable and sustainable productivity.

In the section Enhancing Antibiotic Production by Manipulation of Feedback and Transport, Paragraphs 2, 3, and 4 should be replaced with the following text;

It has been proved that the expression of BGCs was greatly affected by the secretion of end-products, even without toxicity. ActA (ActII-ORF2) and ActB (ActIIORF3), activate the transcription of BGCs in a feed-forward by transportation of the end-products (Tahlan et al., 2007; $\mathrm{Xu}$ et al., 2012). Only one fifth of ACT was produced by the $\operatorname{act} A B$ mutant. There are two waves for ACT production. The expression of key act genes is initially induced by an ACT biosynthetic intermediate. The ACT production is fully induced only when the inner ACT is pumped out.

Overexpression of Avt $\mathrm{AB}$, an $\mathrm{ABC}$ transporter, enhance the production of avermectin $\mathrm{B} 1 \mathrm{a}$ with two-folds. But the production level of oligomycin A, another product from $S$. avermitilis, was found unaltered. The production promotion effects of avtAB could be specific to avermectin in S. avermitilis (Qiu et al., 2011). Co-overexpression of three OTC resistance genes, including $\operatorname{otr} A$ (encoding a ribosomal protection protein), $\operatorname{otr} B$ and $\operatorname{otr} C$ (encoding two efflux proteins), led to $179 \%$ increase of OTC production in Streptomyces rimosus M4018 (Yin et al., 2017).

The biosynthesis of BGCs for the actinobacterial ribosomally synthesized and posttranslationally modified peptides (RiPPs), like planosporicin and microbisporicin, is probably regulated in a feed-forward way. Their production and self-immunity is seemed to be modulated by the multiple $\mathrm{ABC}$ transporter genes in these BGCs (Foulston and Bibb, 2010; Sherwood et al., 2013). GouM, the MFS transporter, is responsible for the secretion of gougerotin outside of Streptomyces graminearus (Wei et al., 2014). The overexpression of Bot $\mathrm{T}$, a putative efflux pump encoded in the bottromycin BGC, increased bottromycin production about 20 times in a heterologous host (Huo et al., 2012).

The authors apologize for these errors and state that this does not change the scientific conclusions of the article in any way. The original article has been updated.
Huang, Y., Yang, D., Pan, G., Tang, G. L., and Shen, B. (2016). Characterization of LnmO as a pathway-specific Crp/Fnr-type positive regulator for leinamycin biosynthesis in Streptomyces atroolivaceus and its application for titer improvement. Appl. Microbiol. Biotechnol. 100, 10555-10562. doi: 10.1007/s00253-016-7864-2

Huo, L., Rachid, S., Stadler, M., Wenzel, S. C., and Muller, R. (2012). Synthetic biotechnology to study and engineer ribosomal bottromycin biosynthesis. Chem. Biol. 19, 1278-1287. doi: 10.1016/j.chembiol.2012.08.013

Jiang, H., and Hutchinson, C. R. (2006). Feedback regulation of doxorubicin biosynthesis in Streptomyces peucetius. Res. Microbiol. 157, 666-674. doi: 10.1016/j.resmic.2006.02.004

Lei, C., Wang, J., Liu, Y., Liu, X., Zhao, G., and Wang, J. (2018). A feedback regulatory model for RifQ-mediated repression of rifamycin export in Amycolatopsis mediterranei. Microb. Cell Fact. 17:14. doi: 10.1186/s12934-018-0863-5

Li, C., He, H., Wang, J., Liu, H., Wang, H., Zhu, Y., et al. (2019). Characterization of a LAL-type regulator NemR in nemadectin biosynthesis and its application 
for increasing nemadectin production in Streptomyces cyaneogriseus. Sci. China Life Sci. 62, 394-405. doi: 10.1007/s11427-018-9442-9

Li, Q., Wang, L., Xie, Y., Wang, S., Chen, R., and Hong, B. (2013). SsaA, a member of a novel class of transcriptional regulators, controls sansanmycin production in Streptomyces sp. strain SS through a feedback mechanism. J. Bacteriol. 195, 2232-2243. doi: 10.1128/jb.00054-13

Li, X., Yu, T., He, Q., McDowall, K. J., Jiang, B., Jiang, Z., et al. (2015). Binding of a biosynthetic intermediate to AtrA modulates the production of lidamycin by Streptomyces globisporus. Mol. Microbiol. 96, 1257-1271. doi: $10.1111 / \mathrm{mmi} .13004$

Liu, G., Tian, Y., Yang, H., and Tan, H. (2005). A pathway-specific transcriptional regulatory gene for nikkomycin biosynthesis in Streptomyces ansochromogenes that also influences colony development. Mol. Microbiol. 55, 1855-1866. doi: $10.1111 / j .1365-2958.2005 .04512 . x$

Liu, Y., Ryu, H., Ge, B., Pan, G., Sun, L., Park, K., et al. (2014). Improvement of Wuyiencin biosynthesis in Streptomyces wuyiensis CK-15 by identification of a key regulator, WysR. J. Microbiol. Biotechnol. 24, 1644-1653. doi: 10.4014/jmb.1405.05017

Ma, D., Wang, C., Chen, H., and Wen, J. (2018). Manipulating the expression of SARP family regulator BulZ and its target gene product to increase tacrolimus production. Appl. Microbiol. Biotechnol. 102, 4887-4900. doi: 10.1007/s00253-018-8979-4

Martin, J.-F., and Liras, P. (2010). Engineering of regulatory cascades and networks controlling antibiotic biosynthesis in Streptomyces. Curr. Opin. Microbiol. 13, 263-273. doi: 10.1016/j.mib.2010.02.008

Mast, Y., Guezguez, J., Handel, F., and Schinko, E. (2015). A complex signaling cascade governs pristinamycin biosynthesis in Streptomyces pristinaespiralis. Appl. Environ. Microbiol. 81, 6621-6636. doi: 10.1128/aem.00728-15

Qiu, J., Zhuo, Y., Zhu, D., Zhou, X., Zhang, L., Bai, L., et al. (2011). Overexpression of the $\mathrm{ABC}$ transporter Avt $\mathrm{AB}$ increases avermectin production in Streptomyces avermitilis. Appl. Microbiol. Biotechnol. 92, 337-345. doi: 10.1007/s00253-011-3439-4

Sherwood, E. J., Hesketh, A. R., and Bibb, M. J. (2013). Cloning and analysis of the planosporicin lantibiotic biosynthetic gene cluster of Planomonospora alba. J. Bacteriol. 195, 2309-2321. doi: 10.1128/jb.02291-12

Sletta, H., Borgos, S. E., Bruheim, P., Sekurova, O. N., Grasdalen, H., Aune, R., et al. (2005). Nystatin biosynthesis and transport: $n y s H$ and $n y s G$ genes encoding a putative ABC transporter system in Streptomyces noursei ATCC 11455 are required for efficient conversion of 10-deoxynystatin to nystatin. Antimicrob. Agents Chemother. 49, 4576-4583. doi: 10.1128/AAC.49.11.4576-4583.2005

Song, K., Wei, L., Liu, J., Wang, J., Qi, H., and Wen, J. (2017). Engineering of the LysR family transcriptional regulator FkbR1 and its target gene to improve ascomycin production. Appl. Microbiol. Biotechnol. 101, 4581-4592. doi: 10.1007/s00253-017-8242-4

Tahlan, K., Ahn, S. K., Sing, A., Bodnaruk, T. D., Willems, A. R., Davidson, A. R., et al. (2007). Initiation of actinorhodin export in Streptomyces coelicolor. Mol. Microbiol. 63, 951-961. doi: 10.1111/j.1365-2958.2006.05 559.x

Wang, L., Tian, X., Wang, J., Yang, H., Fan, K., Xu, G., et al. (2009). Autoregulation of antibiotic biosynthesis by binding of the end product to an atypical response regulator. Proc. Natl. Acad. Sci. U.S.A. 106, 8617-8622. doi: 10.1073/pnas.090059 2106

Wei, J., Tian, Y., Niu, G., and Tan, H. (2014). GouR, a TetR family transcriptional regulator, coordinates the biosynthesis and export of gougerotin in Streptomyces graminearus. Appl. Environ. Microbiol. 80, 714-722. doi: 10.1128/aem.03003-13

Xu, D., Seghezzi, N., Esnault, C., and Virolle, M. J. (2010). Repression of antibiotic production and sporulation in Streptomyces coelicolor by overexpression of a TetR family transcriptional regulator. Appl. Environ. Microbiol. 76, 7741-7753. doi: $10.1128 /$ aem.00819-10

Xu, Y., Willems, A., Au-Yeung, C., Tahlan, K., and Nodwell, J. R. (2012). A twostep mechanism for the activation of actinorhodin export and resistance in Streptomyces coelicolor. MBio. 3, e191-e112.

Yin, S., Wang, W., Wang, X., Zhu, Y., Jia, X., Li, S., et al. (2015). Identification of a cluster-situated activator of oxytetracycline biosynthesis and manipulation of its expression for improved oxytetracycline production in Streptomyces rimosus. Microb. Cell Fact. 14, 46.

Yin, S., Wang, X., Shi, M., Yuan, F., Wang, H., Jia, X., et al. (2017). Improvement of oxytetracycline production mediated via cooperation of resistance genes in Streptomyces rimosus. Sci. China Life Sci. 60, 992-999. doi: 10.1007/s11427-017-9121-4

Zhang, Y., He, H., Liu, H., Wang, H., Wang, X., and Xiang, W. (2016). Characterization of a pathway-specific activator of milbemycin biosynthesis and improved milbemycin production by its overexpression in Streptomyces bingchenggensis. Microb. Cell Fact. 15:152. doi: 10.1186/s12934-016-0552-1

Copyright $\odot 2021 \mathrm{Xia}, \mathrm{Li}, \mathrm{Li}$, Zhan, Mao and Li. This is an open-access article distributed under the terms of the Creative Commons Attribution License (CC BY). The use, distribution or reproduction in other forums is permitted, provided the original author(s) and the copyright owner(s) are credited and that the original publication in this journal is cited, in accordance with accepted academic practice. No use, distribution or reproduction is permitted which does not comply with these terms. 